\title{
Reasoning with Qualified Cardinality Restrictions in Fuzzy Description Logics
}

\author{
Giorgos Stoilos and Giorgos Stamou and Stefanos Kollias
}

\begin{abstract}
Description Logics (DLs) are modern knowledge representation formalisms which are used today in many applications for reasoning with structured knowledge. Moreover, they are used in the Semantic Web (an extension of the current web) through the ontology language OWL. On the other hand fuzzy Description Logics (fuzzy-DLs) have been proposed as expressive logical formalisms capable of capturing and reasoning with vague and imprecise knowledge in the Semantic Web. In the current paper we investigate on the problem of reasoning with qualified cardinality restrictions (QCRs) in fuzzy DLs, extending previous results on simple number restrictions, thus we present a tableaux algorithm for the the fuzzy-DL $\mathbf{f}_{K D}-\mathcal{A L C} \mathcal{L} \mathcal{Q}$.
\end{abstract}

\section{INTRODUCTION}

The Semantic Web [1] has been proposed as an extension of the current web, where information and knowledge would be structured in a machine understandable and processable way. The Semantic Web would be able to automatically carry out complex tasks in a meaningful (semantic) way. In order to represent and reason about structured knowledge in the Semantic Web, W3C has developed and recommended the OWL Web Ontology Language [2]. The logical underpinnings of OWL are mainly very expressive Description Logics (DLs) [3], and more precisely, the $\mathcal{S H O I N}\left(\mathbf{D}^{+}\right)$DL. Description Logics are modern logical formalisms that combine both expressive power as well as decidable and empirically efficient reasoning procedures. In order for applications to publish information on the Semantic Web they have to use OWL and DLs to represent knowledge. Today, DLs and OWL have been used in many applications, like medical [4], image analysis [5], [6], databases [7] and more. This extensive use has highlighted many deficiencies of OWL, which led to requirements for language extensions [4]. One such requirement is the extension with qualified cardinality restrictions [3], [8]. Using such expressive means one is able to create concepts like the visual description of a human as,

$$
\text { Human } \equiv=2 \text { hasPart.Arm } \sqcap=2 \text { hasPart.Leg }
$$

where Human, Arm and Leg are concepts (unary predicates), while hasPart is a role (binary predicates). Such axiom (together with other ones) can be used in a knowledge based image analysis application [5], [6]. In such applications firstly, an image segmentation algorithm is applied, in order to segments an image in various parts (segments) and secondly background knowledge is used in order to annotate

Giorgos Stoilos, Giorgos Stamou and Stefanos Kollias are with the School of Electrical and Computer Engineering, National and Technical University of Athen, Iroon Polytexneiou 9, 15780 Zografou, Greece. For more information abou the paper please contact Giorgos Stoilos: gstoil@image.ece.ntua.gr (label) each segment of the image with a semantic label and thus characterize the content of the segments. Unfortunately, the above axiom cannot be represented with the aid of the OWL language and the $\mathcal{S H O I N}\left(\mathbf{D}^{+}\right) \mathrm{DL}$, but requires the constructor of qualified cardinality restriction $(\mathcal{Q})$, which is not included in the OWL specification.

On the other hand many of the aforementioned applications have special knowledge representational needs, like the requirement to represent and reason about imprecise and vague knowledge. For example, in the image analysis scenario, a segmentation algorithm cannot decide about the membership or non-membership of a segment to a specific concept. Hence, it provides degrees saying for example that segment $o_{2}$ is Green to a degree at least 0.8 or that $o_{2}$ isNear $o_{1}$ to a degree at least 0.75 . For that purpose, fuzzy extensions to Description Logics [9], [10], [11], [12] have been proposed as formalisms capable of representing and reasoning with vague knowledge in Semantic Web enabled applications [13]. Thus, many reasoning algorithm have been presented [9], [14], [15], [16], [12]. On the other hand tableaux based reasoning with qualified cardinality restrictions (QCRs) is missing.

In the current paper we build on the results presented in [12] for simple number restrictions $(\mathcal{N})$, thus presenting an algorithm for reasoning with the fuzzy-DL $\mathrm{f}_{K D}-\mathcal{A L C \mathcal { L }}$. $\mathrm{f}_{K D}-\mathcal{A L C} \mathcal{I} \mathcal{Q}$ extends the well known fuzzy DL $\mathrm{f}_{K D}-\mathcal{A L C}$ [9] with inverse roles and qualified cardinality restrictions (QCRs). A proposal to deal with $\mathrm{f}_{K D}-\mathcal{A L C} \mathcal{Q}$ was first presented in [17], but the procedure was based on a reduction to crisp $\mathcal{A L C Q}$. To the contrary, following [9], [12] we present a direct tableaux algorithm to handle the reasoning problems of $\mathrm{f}_{K D}-\mathcal{A L C I} \mathcal{Q}\left(\mathrm{f}_{K D}-\mathcal{A L C} \mathcal{Q}\right.$ with inverse roles).

The paper is organized as follows. In section II we present the syntax, semantics and the inference services of the fuzzy$\mathrm{DL}_{K D}-\mathcal{A L C} \mathcal{I} \mathcal{Q}$. Subsequently, in section III we investigate on QCRs, in order to be able to provide a sound reasoning procedure. As it is well known in the DL community sound reasoning with QCRs requires some extra care compared to other DL constructors [8]. Then, in section IV we present a reasoning algorithm that decides the key inference problems of the $\mathrm{f}_{K D}-\mathcal{A L C I} \mathcal{Q}$ language. Finally, section $\mathrm{V}$ concludes the paper.

\section{THE FUZZY DL $\mathrm{F}_{K D}-\mathcal{A} \mathcal{L C} \mathcal{I} \mathcal{Q}$}

In this section we introduce the fuzzy DL $\mathrm{f}_{K D}-\mathcal{A L C I} \mathcal{I}$. Following [12] we give such a name to our language in order to indicate the specific fuzzy operators that we are using to provide semantics to it, and more precisely, the Lukasiewicz 
TABLE I

SEMANTICS OF F $K D$ - $\mathcal{A L C I} \mathcal{Q}$-CONCEPTS AND F $K D$ - $\mathcal{A} \mathcal{L C} \mathcal{I} \mathcal{Q}$-ROLES

\begin{tabular}{|c|c|c|}
\hline Constructor & $\overline{\text { Syntax }}$ & Semantics \\
\hline top & $\top$ & $T^{\mathcal{I}}(a)=1$ \\
\hline bottom & $\perp$ & $\perp^{\mathcal{I}}(a)=0$ \\
\hline general negation & $\neg C$ & $(\neg C)^{\mathcal{I}}(a)=1-C^{\mathcal{I}}(a)$ \\
\hline conjunction & $C \sqcap D$ & $(C \sqcap D)^{\mathcal{I}}(a)=\min \left(C^{\mathcal{I}}(a), D^{\mathcal{I}}(a)\right)$ \\
\hline disjunction & $C \sqcup D$ & $(C \sqcup D)^{\mathcal{I}}(a)=\max \left(C^{\mathcal{I}}(a), D^{\mathcal{I}}(a)\right)$ \\
\hline exists restriction & $\exists R . C$ & $(\exists R \cdot C)^{\mathcal{I}}(a)=\sup _{b \in \Delta^{\mathcal{I}}}\left\{\min \left(R^{\mathcal{I}}(a, b), C^{\mathcal{I}}(b)\right)\right\}$ \\
\hline value restriction & $\forall R . C$ & $(\forall R . C)^{\mathcal{I}}(a)=\inf _{b \in \Delta^{\mathcal{I}}}\left\{\max \left(1-R^{\mathcal{I}}(a, b), C^{\mathcal{I}}(b)\right)\right\}$ \\
\hline at-most QCR & $\leq p R \cdot C$ & $\inf _{b_{1}, \ldots, b_{p+1} \in \Delta^{\mathcal{I}}} \max \left(\max _{i=1}^{p+1}\left\{\max \left(1-R^{\mathcal{I}}\left(a, b_{i}\right), 1-C^{\mathcal{I}}\left(b_{i}\right)\right)\right\}, \max _{i<j}\left\{b_{i}=b_{j}\right\}\right)$ \\
\hline at-least QCR & $\geq p R \cdot C$ & $\sup _{b_{1}, \ldots, b_{p} \in \Delta^{\mathcal{I}}} \min \left(\min _{i=1}^{p}\left\{\min \left(R^{\mathcal{I}}\left(a, b_{i}\right), C^{\mathcal{I}}\left(b_{i}\right)\right)\right\}, \min _{i<j}\left\{b_{i} \neq b_{j}\right\}\right)$ \\
\hline inverse roles & $R^{-}$ & $\left(R^{-}\right)^{\mathcal{I}}(b, a)=R^{\mathcal{I}}(a, b)$ \\
\hline
\end{tabular}

complement $(c(a)=1-a)$, the Gödel t-norm $(t(a, b)=$ $\min (a, b))$, the Gödel t-conorm $(u(a, b)=\max (a, b))$ and the Kleene-Dienes fuzzy implication $(\mathcal{J}(a, b)=\max (1-a, b))$.

As usual [3] we have an alphabet of distinct concept names (C), role names $(\mathbf{R})$ and individual names (or simply individuals) (I). $\mathrm{f}_{K D}-\mathcal{A L C} \mathcal{L} \mathcal{Q}$-roles and $\mathrm{f}_{K D}-\mathcal{A L C} \mathcal{L} \mathcal{Q}$-concepts are defined by the following abstract syntax:

$$
\begin{aligned}
C, D \quad:= & \top|\perp| \mathrm{CN}|\neg C| C \sqcap D|C \sqcup D| \\
& \exists R . C|\forall R . C| \geq p R . C \mid \leq p R . C \\
R \quad:= & \operatorname{RN} \mid S^{-}
\end{aligned}
$$

where $\mathrm{CN} \in \mathbf{C}, \mathrm{RN} \in \mathbf{R}$ and $p \in \mathbb{N}$. Note that the syntax $=$ $p R . C$ can be used as a shorthand for $\geq p R . C \sqcap \leq p R . C$. To avoid considering roles $R^{--}$we introduce the function Inv such that $\operatorname{lnv}(R):=\mathrm{RN}$ if $R=R N$, while $\operatorname{lnv}(R):=R N$ if $R=R N^{-}$.

The semantics of fuzzy DLs are provided by a fuzzy interpretation [9]. A fuzzy interpretation consists of a pair $\mathcal{I}=\left(\Delta^{\mathcal{I}},{ }^{\mathcal{I}}\right)$ where the domain $\Delta^{\mathcal{I}}$ is a non-empty set of objects and ${ }^{\mathcal{I}}$ is a fuzzy interpretation function, which maps,

- an individual $a$ to an element of $a^{\mathcal{I}} \in \Delta^{\mathcal{I}}$,

- a concept name $A$ to a membership function $A^{\mathcal{I}}: \Delta^{\mathcal{I}} \rightarrow$ $[0,1]$, and

- a role name $R$ to a membership function $R^{\mathcal{I}}: \Delta^{\mathcal{I}} \times$ $\Delta^{\mathcal{I}} \rightarrow[0,1]$.

Using the fuzzy set theoretic operations, fuzzy interpretations can be extended to interpret $\mathrm{f}_{K D}-\mathcal{A L C \mathcal { L }}$-concepts and roles. The complete set of semantics are depicted in Table I. Most of these semantics have been presented elsewhere [9], [10], [11]. Some remarks are in place for fuzzy QCRs. The semantics of fuzzy QCRs follow the semantics of fuzzy number restrictions first presented in [19] and further revised in [20] to include equalities $(=)$ and inequalities $(\neq)$ of objects of $\Delta^{\mathcal{I}}$ (as depicted in Table I). Although a number of different approaches to qualified number restrictions have been presented in [11], we choose to follow the semantics in [19] since at it was shown in [12], [16] under these semantics and by considering the equalities and inequalities of objects of $\Delta^{\mathcal{I}}$ to be crisp, an efficient procedure to decide the key inference problems of fuzzy DLs can be constructed. This is in contrast to [11] where fuzzy quantification was proposed.

A fuzzy TBox is a finite set of fuzzy concept axioms. Let $A \in \mathbf{C}$ be a concept name and $C$ a $\mathrm{f}_{K D}-\mathcal{A L C} \mathcal{L} \mathcal{Q}$-concept. Fuzzy concept axioms of the form $A \sqsubseteq C$ are called fuzzy concept specialization, while fuzzy concept axioms of the form $A \equiv C$ are called fuzzy concept definitions. As we see, in order not to complicate our presentation, in the present paper we do not address General Concept Inclusion axioms $(C \sqsubseteq D)$. TBoxes without GCIs and where concept names on the left-hand side are uniquely defined are called simple, otherwise are called general. Moreover, we also assume that TBoxes are acyclic. A TBox $\mathcal{T}$ is called acyclic if there is no cyclic dependency among axioms of $\mathcal{T}$. A fuzzy interpretation $\mathcal{I}$ satisfies $A \sqsubseteq C$ if $\forall a \in \Delta^{\mathcal{I}}, A^{\mathcal{I}}(a) \leq C^{\mathcal{I}}(a)$ and it satisfies $A \equiv C$ if $\forall a \in \Delta^{\mathcal{I}}, A^{\mathcal{I}}(a)=C^{\mathcal{I}}(a)$. A fuzzy interpretation satisfies a fuzzy TBox $\mathcal{T}$ if it satisfies all axioms in $\mathcal{T}$. In this case, we say $\mathcal{I}$ is a model of $\mathcal{T}$.

A fuzzy $A B$ ox is a finite set of fuzzy assertions. For $a, b \in$ I, a fuzzy assertion [9] is of the form $(a: C) \bowtie n,((a, b)$ : $R) \bowtie n$, where $\bowtie \in\{\geq,>, \leq,<\}$ or $a \neq b$. We call assertions defined by $\geq$, > positive assertions, while those defined by $\leq,<$ negative assertions. A fuzzy interpretation $\mathcal{I}$ satisfies $(a: C) \geq n((a, b): R \geq n)$ if $C^{\mathcal{I}}\left(a^{\mathcal{I}}\right) \geq n\left(R^{\mathcal{I}}\left(a^{\mathcal{I}}, b^{\mathcal{I}}\right) \geq\right.$ $n$ ) and it satisfies $a \neq b$ if $a^{\mathcal{I}} \neq b^{\mathcal{I}}$; similarly with the other inequalities. A fuzzy interpretation satisfies a fuzzy $\operatorname{ABox} \mathcal{A}$ if it satisfies all fuzzy assertions in $\mathcal{A}$. In this case, we say $\mathcal{I}$ is a model of $\mathcal{A}$. If $\mathcal{A}$ has a model then we say that it is consistent, otherwise it is inconsistent. A fuzzy knowledge base $\Sigma$ is a pair $\langle\mathcal{T}, \mathcal{A}\rangle$, where $\mathcal{T}$ is a fuzzy TBox and $\mathcal{A}$ is a fuzzy ABox.

In the past tableaux reasoning algorithms for fuzzy DLs dealt with both positive and negative fuzzy assertions [9], [10], [12], [18], thus making the algorithms difficult to present and comprehend. In the current paper we will only deal with positive fuzzy assertions. Negative fuzzy assertions of the form $(a: C) \leq n$ and $(a: C)<n$ can be easily transformed into their Positive Inequality Normal Form (PINF), by applying the fuzzy complement in both sides 
of the inequations giving, $(a: \neg C) \geq 1-n$ and $(a$ : $\neg C)>1-n$ (similarly with role assertions), respectively. Please note that the syntax of the language has to be slightly extended in order to allow simple role negation of the form $((a, b): \neg R) \geq 1-n$. Note also that this negation does not effect decidability [9]. In the following, we consider all assertions that exist in a fuzzy $\operatorname{ABox} \mathcal{A}$ to be in their PINF. This idea first appeared in [14] but it was not used for tableaux algorithms, thus many definitions [9], [10], [16] will be revised here.

In the following we use the symbol $\triangleright$ as a placeholder for the inequalities $\geq$, $>$. Finally, we use the symbol + to denote the strengthening or weakening of an inequality. For example applying + to $\geq$ gives $>$, i.e. strengthens the inequality, while applying it to $>$ gives $\geq$, i.e. weakens the inequality.

Following [9], we introduce the concept of conjugated pairs of fuzzy assertions, to represent pairs of assertions that form a contradiction. In the presence of only PINF assertions and inverse roles the definition of conjugated pairs has to be changed from the ones in [9], [10], [18] in order to cover these new features. Let $\phi$ be a crisp $\mathcal{A L C \mathcal { C }}$ assertion. Then $\neg \phi$ represents the same assertion but for the negative concept or role. For example if $\phi=(a, b): R$, then $\neg \phi=(a, b): \neg R$. The possible conjugated pairs are defined in In the presence

TABLE II

CONJUGATED PAIRS OF FUZZY ASSERTIONS

\begin{tabular}{l|c|c}
\hline \hline & $\neg \phi>m$ & $\neg \phi \geq m$ \\
\hline$\phi \geq n$ & $n+m \geq 1$ & $n+m>1$ \\
$\phi>n$ & $n+m \geq 1$ & $n+m \geq 1$ \\
\hline \hline
\end{tabular}

of inverse roles one should also take under consideration possible inverse roles when checking for conjugation two fuzzy role assertions. For example, the assertion $((a, b)$ : $R)>0.9$, conjugates with $((b, a): \neg \operatorname{lnv}(R)) \geq 0.1$.

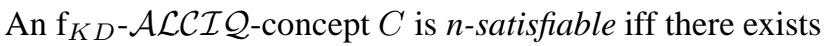
some fuzzy interpretation $\mathcal{I}$ for which there is some $a \in \Delta^{\mathcal{I}}$ such that $C^{\mathcal{I}}(a)=n$, and $n \in(0,1]$. A fuzzy knowledge base $\Sigma$ is satisfiable iff there exists a fuzzy interpretation $\mathcal{I}$ which satisfies all axioms in $\Sigma$. Furthermore, a concept $C$ is subsumed by a concept $D$ w.r.t. $\mathcal{T}$ if for all models $\mathcal{I}$ of $\mathcal{T}$, $\forall a \in \Delta^{\mathcal{I}}, C^{\mathcal{I}}(a) \leq D^{\mathcal{I}}(a)$. Finally, for $\phi$ a fuzzy assertion or a concept subsumption, $\Sigma$ entails $\Psi$, written $\Sigma \models \Psi$ iff any model of $\Sigma$ also satisfies $\Psi$.

The problems of concept $n$-satisfiability, subsumption and entailment w.r.t. a knowledge base $\Sigma$ can be reduced to the problem of fuzzy ABox consistency w.r.t. a TBox $\mathcal{T}$ [9]. More precisely, a concept $C$ is $n$-satisfiable w.r.t. $\mathcal{T}$ iff $\{(a$ : $C) \geq n\}$ is consistent, w.r.t. $\mathcal{T}$. Moreover, for $\Sigma=\langle\mathcal{T}, \mathcal{A}\rangle$, and a PINF assertion $\phi \triangleright n, \Sigma \models \phi \triangleright n$ iff $\Sigma=\langle\mathcal{T}, \mathcal{A} \cup\{\neg \phi+$ $\triangleright 1-n\}\rangle$ is unsatisfiable. In [9] it was shown that $\Sigma \models \phi \geq n$ if and only if $\Sigma=\langle\mathcal{T}, \mathcal{A} \cup\{\phi<n\}\rangle$ is unsatisfiable. Since we are only considering PINF fuzzy assertions we transform $\phi<n$, into its PINF form, $\neg \phi>1-n$, and similarly with $>$. Furthermore, the subsumption of two fuzzy concepts $C$ and
$D$ w.r.t. $\Sigma$ can also be reduced to $\mathrm{ABox}$ consistency w.r.t. $\mathcal{T}$. More formally, $\Sigma \models C \sqsubseteq D$ iff $\langle\mathcal{T}, \mathcal{A} \cup\{(a: C) \geq n,(a$ : $\neg D)>1-n\}\rangle$, for both $n \in\left\{n_{1}, n_{2}\right\}, n_{1} \in(0,0.5]$ and $n_{2} \in(0.5,1]$, is unsatisfiable [9], where again the negative assertions in [9] have been transformed into their PINF. In the presence of only simple and acyclic TBoxes, the above problems can be further reduced to ABox consistency w.r.t. an empty TBox [3]. Thus, without loss of generality, in the following we will assume that TBoxes are empty.

When reasoning with $\mathrm{f}_{K D}$-DLs a very important notion is that of a witnessed model [21]. As one can depict from Table I the semantics of existential restrictions are defined in terms of the supremum. In a witnessed model if $(\exists R . C)^{\mathcal{I}}(a)=n$ then there is some $b \in \Delta^{\mathcal{I}}$ such that either $R^{\mathcal{I}}\left(a, b_{i}\right)=n$ or $C^{\mathcal{I}}\left(b_{i}\right)=n$, i.e. there is some $b \in \Delta^{\mathcal{I}}$ that witnesses the membership degree of $a$ to $\exists R . C$, and sup is really max. Hence, from a fuzzy assertion $(a: \exists R . C) \geq n$ we can conclude that there exists some $b$ such that $(a, b): R \geq n$ and $(b: C) \geq n$. In the following and without loss of generality we assume that all models are witnessed.

\section{QuAlified CARDinAlity RESTRICTIONS IN F-DLS}

Stoilos et. al. [12] proved that reasoning w.r.t. the semantics of number restrictions, first proposed in [19] (see also Table I), can be reduced to a simple counting problem of $R$ instance relations between individuals. More precisely, an inequality of the form $(\geq p R)^{\mathcal{I}}(a) \geq n$ intuitively means that there are at-least $p$ objects $b_{i} \in \Delta^{\mathcal{I}}$ such that $R^{\mathcal{I}}\left(a, b_{i}\right) \geq n$, with $1 \leq i \leq p$. On the other hand $(\leq p R)^{\mathcal{I}}(a) \geq n$ means that there are at-most $p$ objects $b_{i} \in \Delta^{\mathcal{I}}$ such that $R^{\mathcal{I}}\left(a, b_{i}\right)>1-n$. One can then translate these propositions to tableaux reasoning rules [12]. For example, if one has $(a: \leq p R) \geq n$ and there are $p+1$ individuals $b_{i}$ such that $\left(a, b_{i}\right): R \geq k_{i}$ with $k_{i}>1-n$, then the algorithm should non-deterministically merge pairs of $b_{i}$. These results can be trivially extended to the case of qualified cardinality restrictions. More precisely, $(\leq p R . C)^{\mathcal{I}}(a) \geq n$ means that there are at-most $p$ objects $b_{i} \in \Delta^{\mathcal{I}}$ such that $R^{\mathcal{I}}\left(a, b_{i}\right)>1-n$ and $C^{\mathcal{I}}\left(b_{i}\right) \geq 1-n$. Thus, the tableaux expansion rules from [12] need to be extended accordingly to provide reasoning for QCRs.

But additionally, in order to provide sound reasoning for QCRs, classical DL reasoning procedures use a special rule, called choose-rule [22]. This rule inserts implied semantic information during the reasoning process, which is vital for the application of the rules that are relative to the qualified cardinality restrictions. Consider for example the fuzzy ABox, $\mathcal{A}_{c}=\{(a:(\geq 3 R . \top)) \geq 0.7,(a:(\leq$ $1 R . B)) \geq 0.7,(a:(\leq 1 R . \neg B)) \geq 0.7\}$ taken from crisp DLs [8] and adapted to the fuzzy case. The above ABox is inconsistent because the following conditions must hold:

$$
\begin{aligned}
& \sup _{b_{1}, \ldots, b_{3} \in \Delta^{\mathcal{I}}} \min _{i=1}^{3}\left\{\min \left(R^{\mathcal{I}}\left(a, b_{i}\right), \top^{\mathcal{I}}\left(b_{i}\right)\right)\right\} \geq 0.7, \\
& \inf _{b_{1}, b_{2} \in \Delta^{\mathcal{I}}} \max _{i=1}^{2}\left\{\max \left(1-R^{\mathcal{I}}\left(a^{\mathcal{I}}, b_{i}\right), 1-B^{\mathcal{I}}\left(b_{i}\right)\right)\right\} \geq 0.7,
\end{aligned}
$$




$$
\inf _{b_{1}, b_{2} \in \Delta^{\mathcal{I}}} \max _{i=1}^{2}\left\{\max \left(1-R^{\mathcal{I}}\left(a^{\mathcal{I}}, b_{i}\right), B^{\mathcal{I}}\left(b_{i}\right)\right)\right\} \geq 0.7 .
$$

From the first equation we get that there must exist three $b_{i} \in \Delta^{\mathcal{I}}$ such that $\forall b_{i}, R^{\mathcal{I}}\left(a^{\mathcal{I}}, b_{i}\right) \geq 0.7$, with $1 \leq i \leq 3$, while from the last two we get that for each two objects $b_{i}$ and $b_{j}$, it holds that

$$
\max \left(1-R^{\mathcal{I}}\left(a^{\mathcal{I}}, b_{i}\right), 1-B^{\mathcal{I}}\left(b_{i}\right)\right) \geq 0.7
$$

and

$$
\max \left(1-R^{\mathcal{I}}\left(a^{\mathcal{I}}, b_{j}\right), B^{\mathcal{I}}\left(b_{j}\right)\right) \geq 0.7, \text { for } 1 \leq i<j \leq 3 .
$$

Since from the first restriction we know that $\forall b_{i}, 1-$ $R^{\mathcal{I}}\left(a^{\mathcal{I}}, b_{i}\right) \leq 0.3<0.7$, then for every two objects $b_{i}$ and $b_{j}$ for one of the two, say $b_{i}$, it should hold that, $1-B^{\mathcal{I}}\left(b_{i}\right) \geq 0.7$ while for $b_{j}$ it should hold that $B^{\mathcal{I}}\left(b_{j}\right) \geq$ $0.7 \Rightarrow 1-B^{\mathcal{I}}\left(b_{j}\right) \leq 0.3<0.7$. Obviously, this cannot happen for every pair of objects $\left\langle b_{i}, b_{j}\right\rangle, 1 \leq i<j \leq 3$.

However, applying a naive algorithm could lead to incorrect results. More precisely, suppose that first, in order to satisfy the assertion $(a:(\geq 3 R . \top)) \geq 0.7$ we create three individuals $b_{i}$ for which $R\left(a, b_{i}\right) \geq 0.7,1 \leq i \leq 3$. Then, it would appear as we have satisfied all assertions of the ABox since there is no obvious contradiction (clash) within the ABox. This means that the algorithm is unsound. As noted in [8], the reason for this mistake is that there exists implied information that is not taken under consideration by the above algorithm. In the crisp case the implied information is that any individual $b_{i}$ either belongs to $B$ or to $\neg B$. Such an analysis is often referred to as reasoning by cases. In the case of fuzzy DLs, as it was noted in [18], we have that for all $n \in[0,1]$, for all individuals $b_{i} \in \mathbf{I}$ and for all $\mathrm{f}_{K D}-\mathcal{A L C I} \mathcal{Q}$-concepts $B$ either $B\left(b_{i}\right)>n$ or $B\left(b_{i}\right) \leq n .{ }^{1}$ Hence, in our case we should have that either $B\left(b_{i}\right)>0.3$ or $B\left(b_{i}\right) \leq 0.3\left(\neg B\left(b_{i}\right) \geq 0.7\right.$ in PINF), for all $1 \leq i \leq 3$. If we choose $B\left(b_{i}\right)>0.3$ for two out of the three individuals $b_{i}$, then the first at-most restriction is violated, since it requires that $B^{\mathcal{I}}\left(b_{i}\right) \leq 0.3$, while if we choose $\neg B\left(b_{i}\right) \geq 0.7$, then $1-B\left(b_{i}\right) \geq 0.7>0.3$ and thus, the second atmost restriction is violated. We conclude that in both cases the assertions cannot be satisfied simultaneously, hence the ABox is inconsistent.

In order to construct a correct algorithm we should perform such reasoning by cases to add any implied information during the construction of the model. This analysis is performed by the special rule called choose-rule which adds information that is relevant to the application (or not) of at-most QCRs. Based on the analysis we presented in the beginning of this section, we can see that for a fuzzy assertion $(a: \leq p R . C) \geq n$, the relevant information is the inequality $+\geq=>$ and the degree $1-n$; similarly for a fuzzy assertion $(a: \leq p R . C)>n$. For example, in the previous case we used the degrees $1-0.7=0.3$ and $1-0.3=0.7$ (due to PINF), as well as the inequality " $>$ ". In other words these values are the threshold for either satisfying or not a fuzzy assertion that involves QCRs.

\footnotetext{
${ }^{1}$ Note that in [18] the authors actually use the case analysis "either $B\left(b_{i}\right) \geq n$ or $B\left(b_{i}\right)<n$ ". Obviously, both these forms are equivalent.
}

\section{The Tableaux Algorithm}

In section II we show that all inference problems of fuzzy DLs can be reduced to the problem of consistency checking for ABoxes. Consistency is usually checked with tableaux algorithms that try to construct a fuzzy tableau for $\mathcal{A}$. Given the notion of a fuzzy tableau, it is quite straightforward to prove the algorithm is a decision procedure for ABox consistency. Our definition of a fuzzy tableau follows those presented for the fuzzy DLs $\mathrm{f}_{K D^{-}} \mathcal{S} \mathcal{I}$ and $\mathrm{f}_{K D^{-}} \mathcal{S} \mathcal{H} \mathcal{I} \mathcal{N}$ [12], but on the one hand is reduced in the sense that we do not handle transitive roles and role hierarchies, while on the other hand some properties from [12] are extended in order to handle QCRs.

Without loss of generality, we assume all concepts $C$ occurring in assertions $(a: C) \triangleright n \in \mathcal{A}$ to be in their negation normal form (NNF) [23]; i.e., negations occur in front of concept names only. An $\mathrm{f}_{K D}-\mathcal{A L C I} \mathcal{L}$-concept can be transformed into an equivalent one in NNF by pushing negations inwards making use of the De Morgan laws, which in our case are satisfied, as well as the following concept equivalences:

$$
\begin{aligned}
\neg \exists R . C & \equiv \forall R .(\neg C), \quad \neg \forall R . C \quad \equiv \exists R .(\neg C), \\
\neg \leq p_{1} R . C & \equiv \geq\left(p_{1}+1\right) R . C, \\
\neg \geq p_{1} R . C & \equiv \begin{cases}\leq\left(p_{1}-1\right) R . C, & p_{1} \in \mathbb{N}^{*} \\
\perp, & p_{1}=0\end{cases}
\end{aligned}
$$

Definition 4.1: For a concept $D$ we inductively define the set of sub-concepts $(\operatorname{sub}(D))$ as,

$$
\begin{aligned}
\operatorname{sub}(A) & =\{A\} \text { for every atomic concept } A \in \mathbf{C}, \\
\operatorname{sub}(C \sqcap D) & =\{C \sqcap D\} \cup\{\operatorname{sub}(C)\} \cup\{\operatorname{sub}(D)\}, \\
\operatorname{sub}(C \sqcup D) & =\{C \sqcup D\} \cup\{\operatorname{sub}(C)\} \cup\{\operatorname{sub}(D)\}, \\
\operatorname{sub}(\exists R . C) & =\{\exists R . C\} \cup\{\operatorname{sub}(C)\}, \\
\operatorname{sub}(\forall R . C) & =\{\forall R . C\} \cup\{\operatorname{sub}(C)\}, \\
\operatorname{sub}(\geq n R . C) & =\{\geq n R . C\} \text { and } \\
\operatorname{sub}(\leq n R . C) & =\{\leq n R . C\} \cup\{\operatorname{sub}(C)\}
\end{aligned}
$$

Definition 4.2: For a fuzzy concept $D$ and an RBox $\mathcal{R}$ we define $\operatorname{clos}(D, \mathcal{R})$ as the smallest set of $\mathrm{f}_{K D^{-}} \mathcal{S H O I} \mathcal{Q}$ concepts which satisfies the following:

- $D \in \operatorname{clos}(D, \mathcal{R})$,

- $\operatorname{clos}(D, \mathcal{R})$ is closed under subconcepts of $D$ and $\sim D$, and

- if $\forall R . C \in \operatorname{clos}(D, \mathcal{R})$ and $\operatorname{Trans}(R)$, then $\forall R . C \in$ $\operatorname{clos}(D, \mathcal{R})$

Finelly we define $\operatorname{clos}(\mathcal{A}, \mathcal{R})=\bigcup_{(a: D) \triangleright n \in \mathcal{A}} \operatorname{clos}(D, \mathcal{R}) . \quad \diamond$

When $\mathcal{R}$ is clear from the context we simply write $\operatorname{clos}(\mathcal{A})$.

Definition 4.3: If $\mathcal{A}$ is an $\mathrm{f}_{K D}-\mathcal{A L C} \mathcal{L} \mathcal{Q}$ ABox, $\mathbf{R}_{\mathcal{A}}$ is the set of roles occurring in $\mathcal{A}$ together with their inverses and $\mathbf{I}_{\mathcal{A}}$ is the set of individuals in $\mathcal{A}$, a fuzzy tableau $T$ for $\mathcal{A}$ is defined to be a quadruple $(\mathbf{S}, \mathcal{L}, \mathcal{E}, \mathcal{V})$ such that: $\mathbf{S}$ is a set of elements, $\mathcal{L}: \mathbf{S} \times \operatorname{clos}(\mathcal{A}) \rightarrow[0,1]$ maps each element and concept, that is a subset of $\operatorname{clos}(\mathcal{A})$, to the membership degree of that element to the concept, $\mathcal{E}: \mathbf{R}_{\mathcal{A}} \times \mathbf{S} \times \mathbf{S} \rightarrow$ 
$[0,1]$ maps each role of $\mathbf{R}_{\mathcal{A}}$ and pair of elements to the membership degree of the pair to the role, and $\mathcal{V}: \mathbf{I}_{\mathcal{A}} \rightarrow \mathbf{S}$ maps individuals occurring in $\mathcal{A}$ to elements of $\mathbf{S}$. For all $s, t \in \mathbf{S}, C, E \in \operatorname{clos}(\mathcal{A}), n \in[0,1]$ and $R \in \mathbf{R}_{\mathcal{A}}, T$ satisfies:

1) $\mathcal{L}(s, \perp)=0$ and $\mathcal{L}(s, \top)=1$ for all $s \in \mathbf{S}$,

2) If $\mathcal{L}(s, \neg A)=n$, then $\mathcal{L}(s, A)=1-n$,

3) If $\mathcal{E}(\neg R,\langle s, t\rangle)=n$, then $\mathcal{E}(R,\langle s, t\rangle)=1-n$,

4) If $\mathcal{L}(s, C \sqcap E) \triangleright n$, then $\mathcal{L}(s, C) \triangleright n$ and $\mathcal{L}(s, E) \triangleright n$,

5) If $\mathcal{L}(s, C \sqcup E) \triangleright n$, then $\mathcal{L}(s, C) \triangleright n$ or $\mathcal{L}(s, E) \triangleright n$,

6) If $\mathcal{L}(s, \forall R . C) \triangleright n$, then either $\mathcal{E}(\neg R,\langle s, t\rangle) \triangleright n$ or $\mathcal{L}(t, C) \triangleright n$

7) If $\mathcal{L}(s, \exists R . C) \triangleright n$, then there exists $t \in \mathbf{S}$ such that $\mathcal{E}(R,\langle s, t\rangle) \triangleright n$ and $\mathcal{L}(t, C) \triangleright n$,

8) $\mathcal{E}(R,\langle s, t\rangle) \triangleright n$ iff $\mathcal{E}(\operatorname{Inv}(R),\langle t, s\rangle) \triangleright n$,

9) If $\mathcal{L}(s, \geq p R . C) \triangleright n$, then $\sharp R^{T}(s, \triangleright, n, C) \geq p$,

10) If $\mathcal{L}(s, \leq p R . C) \triangleright n$, then $\sharp R^{T}(s,+\triangleright, 1-n, C) \leq p$,

11) If $\mathcal{L}(s, \leq p R . C) \triangleright n$ and $\mathcal{E}(R,\langle s, t\rangle)+\triangleright 1-n$, then either $\mathcal{L}(t, \neg C) \triangleright n$ or $\mathcal{L}(t, C)+\triangleright 1-n$,

12) If $(a: C) \triangleright n \in \mathcal{A}$, then $\mathcal{L}(\mathcal{V}(a), C) \triangleright n$,

13) If $((a, b): R) \triangleright n \in \mathcal{A}$, then $\mathcal{E}(R,\langle\mathcal{V}(a), \mathcal{V}(b)\rangle) \triangleright n$,

14) If $a \neq b \in \mathcal{A}$, then $\mathcal{V}(a) \neq \mathcal{V}(b)$.

where $\sharp$ denotes the cardinality of a set and $R^{T}(s, \triangleright, n, C)=$ $\{t \in \mathbf{S} \mid \mathcal{E}(R,\langle s, t\rangle) \triangleright n$ and $\mathcal{L}(s, C) \triangleright n\}$ returns the set of elements $t \in \mathbf{S}$ that participate in $R$ with the element $s$ and at the same time to the concept $C$ with a degree, greater or equal or greater than $n$.

Lemma 4.4: An $\mathrm{f}_{K D}-\mathcal{A L C I} \mathcal{L}$ ABox $\mathcal{A}$ is consistent iff there exists a fuzzy tableau for $\mathcal{A}$.

Proof: [Sketch] For the if direction if $T=(\mathbf{S}, \mathcal{L}, \mathcal{E}, \mathcal{V})$ is a fuzzy tableau for $\mathcal{A}$, we can construct a fuzzy interpretation $\mathcal{I}=\left(\Delta^{\mathcal{I}},{ }^{\mathcal{I}}\right)$ that is a model of $\mathcal{A}$ as follows:

$$
\begin{aligned}
\Delta^{\mathcal{I}} & =\mathbf{S} \\
a^{\mathcal{I}} & =\mathcal{V}(a), a \text { occurs in } \mathcal{A} \\
\top^{\mathcal{I}}(s) & =\mathcal{L}(s, \top), \text { for all } s \in \mathbf{S} \\
\perp^{\mathcal{I}}(s) & =\mathcal{L}(s, \perp), \text { for all } s \in \mathbf{S} \\
A^{\mathcal{I}}(s) & =\mathcal{L}(s, A) \text { for all } s \in \mathbf{S} \\
R^{\mathcal{I}}(s, t) & =\mathcal{E}(R,\langle s, t\rangle) \text { for all }\langle s, t\rangle \in \mathbf{S} \times \mathbf{S}
\end{aligned}
$$

To prove that $\mathcal{I}$ is a model of $\mathcal{A}$, we can show by induction on the structure of concepts that $\mathcal{L}(s, C) \triangleright n$ implies $C^{\mathcal{I}}(s) \triangleright n$ for all $s \in \mathbf{S}$. Together with properties 12, 13, 14 and the interpretation of individuals and roles, this implies that $\mathcal{I}$ satisfies each fuzzy assertion in $\mathcal{A}$.

For the converse, if $\mathcal{I}=\left(\Delta^{\mathcal{I}},{ }^{\mathcal{I}}\right)$ is a model of $\mathcal{A}$, then a fuzzy tableau $T=(\mathbf{S}, \mathcal{L}, \mathcal{E}, \mathcal{V})$ for $\mathcal{A}$ can be defined as follows:

$$
\begin{aligned}
\mathbf{S} & =\Delta^{\mathcal{I}} \\
\mathcal{E}(R,\langle s, t\rangle) & =R^{\mathcal{I}}(s, t) \\
\mathcal{L}(s, C) & =C^{\mathcal{I}}(s) \\
\mathcal{V}(a) & =a^{\mathcal{I}}
\end{aligned}
$$

It is easy to show that $T$ is a fuzzy tableau for $\mathcal{A}$.

The above lemma suggests that in order to decide the key inference problems of $\mathrm{f}_{K D}-\mathcal{A L C \mathcal { Q }}$ we can develop an algorithm that constructs a fuzzy tableau for $\mathrm{f}_{K D}-\mathcal{A L C \mathcal { Q }}$.

Definition 4.5: A completion-forest $\mathcal{F}$ for an $\mathrm{f}_{K D}-\mathcal{A L C} \mathcal{L} \mathcal{Q}$ ABox $\mathcal{A}$ is a collection of trees whose distinguished roots are arbitrarily connected by edges. Each node $x$ is labelled with a set $\{\langle C, \triangleright, n\rangle\} \subseteq \mathcal{L}(x)$, where $C \in \operatorname{clos}(\mathcal{A})$ and $n \in[0,1]$. Each edge $\langle x, y\rangle$ is labelled with a set $\{\langle R, \triangleright, n\rangle\} \subseteq \mathcal{L}(\langle x, y\rangle)$, where $R \in \mathbf{R}_{\mathcal{A}}$ are (possibly inverse) roles occurring in $\mathcal{A}$. Intuitively, each triple $\langle C, \triangleright, n\rangle \quad(\langle R, \triangleright, n\rangle)$, called membership triple, represents the membership degree and the type of assertion of each node (pair of nodes) to a concept $C$ (role $R$ ).

If nodes $x$ and $y$ are connected by an edge $\langle x, y\rangle$ with $\langle R, \triangleright, n\rangle \in \mathcal{L}(\langle x, y\rangle)$, then $y$ is called an $R_{\triangleright n}$-successor of $x$ and $x$ is called an $R_{\triangleright n}$-predecessor of $y$. If $y$ is an $R_{\triangleright n}$-successor or an $\operatorname{lnv}(R)_{\triangleright n}$-predecessor of $x$, then $y$ is called an $R_{\triangleright n}$-neighbour of $x$. Let $y$ be an $R_{>n}$-neighbour of $x$, the edge $\langle x, y\rangle$ is conjugated with triples $\langle\neg R, \triangleright, m\rangle$ if $n+m \geq 1$. Similarly, we can extend it to the case of $R_{\geq n}$-neighbours. As usual, ancestor is the transitive closure of predecessor.

For a role $R$, a concept $C$, a node $x$ in $\mathcal{F}$, an inequality type $\triangleright$ and a membership degree $n \in[0,1]$ we define: $R_{c}^{\mathcal{F}}(x, \triangleright, n, C)=\left\{y \mid y\right.$ is an $R_{\triangleright^{\prime} n^{\prime}}$-neighbour of $x$, $\langle x, y\rangle$ is conjugated with $\langle\neg R, \triangleright, n\rangle$ and $\mathcal{L}(y) \cup\{\langle\neg C, \triangleright, n\rangle\}$ contains a clash\}.

For a node $x, \mathcal{L}(x)$ is said to contain a clash if it contains one of the following:

- two conjugated pairs of triples,

- one of $\langle\perp, \geq, n\rangle$, with $n>0,\langle\perp\rangle, n$,$\rangle or \langle C,>, 1\rangle$, or

- some triple $\langle\leq p R . C, \triangleright, n\rangle$ and $x$ has $p+1 R_{\triangleright^{\prime} n_{i}}{ }^{-}$ neighbours $y_{0}, \ldots, y_{p}$, all $\left\langle x, y_{i}\right\rangle$ are conjugated with $\langle\neg R, \triangleright, n\rangle, \mathcal{L}\left(y_{i}\right) \cup\{\langle\neg C, \triangleright, n\rangle\}$ contains a clash and $y_{i} \neq y_{j}, n_{i}, n \in[0,1]$, for all $0 \leq i<j \leq p$

Moreover, for an edge $\langle x, y\rangle, \mathcal{L}(\langle x, y\rangle)$ is said to contain a clash if there exist two conjugated triples in $\mathcal{L}(\langle x, y\rangle)$, if $\langle R,>, 1\rangle \in \mathcal{L}(\langle x, y\rangle)$ or if $\mathcal{L}(\langle x, y\rangle) \cup\{\langle\operatorname{lnv}(R), \triangleright, n\rangle \mid$ $\langle R, \triangleright, n\rangle \in \mathcal{L}(\langle y, x\rangle)\}$, where $x, y$ are root nodes, contains two conjugated triples.

For an $\mathrm{f}_{K D}-\mathcal{A L C} \mathcal{I} \mathcal{Q}$ B Box $\mathcal{A}$, the algorithm initializes a forest $\mathcal{F}$ to contain

i a root node $x_{a_{i}}$, for each individual $a_{i} \in \mathbf{I}_{\mathcal{A}}$ occurring in the $\operatorname{ABox} \mathcal{A}$, labelled with $\mathcal{L}\left(x_{a_{i}}\right)$ such that $\langle C, \triangleright, n\rangle \in \mathcal{L}\left(x_{a_{i}}\right)$ for each assertion of the form $\left(a_{i}: C\right) \triangleright n$ in $\mathcal{A}$,

ii an edge $\left\langle x_{a_{i}}, x_{a_{j}}\right\rangle$, for each assertion $\left(\left\langle a_{i}, a_{j}\right\rangle: R\right) \triangleright n$ in $\mathcal{A}$, labelled with $\mathcal{L}\left(\left\langle x_{a_{i}}, x_{a_{j}}\right\rangle\right)$ such that $\langle R, \triangleright, n\rangle \in$ $\mathcal{L}\left(\left\langle x_{a_{i}}, x_{a_{j}}\right\rangle\right)$, and

iii the relation $\neq$ as $x_{a_{i}} \neq x_{a_{j}}$ if $a_{i} \neq a_{j} \in \mathcal{A}$ and the relation $\doteq$ to be empty.

$\mathcal{F}$ is then expanded by repeatedly applying the completion rules from Table III. The completion forest is complete when, for some node $x, \mathcal{L}(x)$ contains a clash, or none of the 
TABLE III

TABLEAUX EXPANSION RULES FOR $\mathrm{F}_{K} D-\mathcal{A} \mathcal{L} \mathcal{C} \mathcal{I} \mathcal{Q}$

\begin{tabular}{|c|c|c|}
\hline Rule & & Description \\
\hline$\left(\Pi_{\triangleright}\right)$ & $\begin{aligned} & \text { if } 1 . \\
& 2 . \\
& \text { then }\end{aligned}$ & $\begin{array}{l}\left\langle C_{1} \sqcap C_{2}, \triangleright, n\right\rangle \in \mathcal{L}(x) \text { and } \\
\left\{\left\langle C_{1}, \triangleright, n\right\rangle,\left\langle C_{2}, \triangleright, n\right\rangle\right\} \nsubseteq \mathcal{L}(x) \\
\mathcal{L}(x) \rightarrow \mathcal{L}(x) \cup\left\{\left\langle C_{1}, \triangleright, n\right\rangle,\left\langle C_{2}, \triangleright, n\right\rangle\right\}\end{array}$ \\
\hline$\left(\sqcup_{\triangleright}\right)$ & $\begin{aligned} & \text { if } 1 . \\
& 2 . \\
& \text { then }\end{aligned}$ & $\begin{array}{l}\left\langle C_{1} \sqcup C_{2}, \triangleright, n\right\rangle \in \mathcal{L}(x), x \text { and } \\
\left\{\left\langle C_{1}, \triangleright, n\right\rangle,\left\langle C_{2}, \triangleright, n\right\rangle\right\} \cap \mathcal{L}(x)=\emptyset \\
\mathcal{L}(x) \rightarrow \mathcal{L}(x) \cup\{C\} \text { for some } C \in\left\{\left\langle C_{1}, \triangleright, n\right\rangle,\left\langle C_{2}, \triangleright, n\right\rangle\right\}\end{array}$ \\
\hline$\left(\exists_{\triangleright}\right)$ & $\begin{aligned} & \text { if } 1 . \\
& 2 . \\
& \text { then }\end{aligned}$ & $\begin{array}{l}\langle\exists R . C, \triangleright, n\rangle \in \mathcal{L}(x), \\
x \text { has no } R_{\triangleright} n \text {-neighbour } y \text { with }\langle C, \triangleright, n\rangle \in \mathcal{L}(y) \\
\text { create a new node } y \text { with } \mathcal{L}(\langle x, y\rangle)=\{\langle R, \triangleright, n\rangle\}, \mathcal{L}(y)=\{\langle C, \triangleright, n\rangle\},\end{array}$ \\
\hline$\left(\forall_{\triangleright}\right)$ & $\begin{array}{r}\text { if } 1 . \\
2 . \\
3 . \\
\text { then }\end{array}$ & $\begin{array}{l}\langle\forall R . C, \triangleright, n\rangle \in \mathcal{L}(x), \\
x \text { has an } R_{\triangleright} n_{n} \text {-neighbour } y \text { with }\langle C, \triangleright, n\rangle \notin \mathcal{L}(y) \text { and } \\
\langle\neg R, \triangleright, n\rangle \text { is conjugated with the edge }\langle x, y\rangle \\
\mathcal{L}(y) \rightarrow \mathcal{L}(y) \cup\{\langle C, \triangleright, n\rangle\}\end{array}$ \\
\hline (choose) & $\begin{array}{r}\text { if } 1 . \\
2 . \\
3 . \\
\text { then }\end{array}$ & $\begin{array}{l}\langle\leq p R . C, \triangleright, n\rangle \in \mathcal{L}(x), \\
\text { there is an } R \text {-neighbour } y \text { of } x, \text { with }\{\langle\sim C, \triangleright, n\rangle,\langle C,+\triangleright, 1-n\rangle\} \cap \mathcal{L}(x)=\emptyset \text {, and } \\
\langle x, y\rangle \text { conjugates with }\langle\neg R, \triangleright, n\rangle \\
\mathcal{L}(y) \rightarrow \mathcal{L}(y) \cup\{E\}, \text { for some } E \in\{\langle\sim C, \triangleright, n\rangle,\langle C,+\triangleright, 1-n\rangle\}\end{array}$ \\
\hline$\left(\geq_{\triangleright}\right)$ & $\begin{array}{l}\text { if } 1 . \\
2 . \\
\text { then }\end{array}$ & $\begin{array}{l}\langle\geq p R . C, \triangleright, n\rangle \in \mathcal{L}(x) \\
\text { there are no } p R_{\triangleright n} \text {-neighbours } y_{1}, \ldots, y_{p} \text { of } x \text { with }\langle C, \triangleright, n\rangle \in \mathcal{L}\left(y_{i}\right) \\
\text { and } y_{i} \neq y_{j} \text { for } 1 \leq i<j \leq p \\
\text { create } p \text { new nodes } y_{1}, \ldots, y_{p}, \text { with } \mathcal{L}\left(\left\langle x, y_{i}\right\rangle\right)=\{\langle R, \triangleright, n\rangle\} \\
\mathcal{L}\left(y_{i}\right)=\{\langle C, \triangleright, n\rangle\} \text { and } y_{i} \neq y_{j} \text { for } 1 \leq i<j \leq p\end{array}$ \\
\hline$\left(\leq_{\triangleright}\right)$ & $\begin{array}{l}\text { if } 1 . \\
2 . \\
\text { then }\end{array}$ & $\begin{array}{l}\langle\leq p R . C, \triangleright, n\rangle \in \mathcal{L}(x) \\
\sharp R_{c}^{\mathcal{F}}(x, \triangleright, n, C)>p \text {, there are two of them } y, z \text {, with no } y \neq z \text { and } \\
y \text { is neither a root node nor an ancestor of } z \\
\text { 1. } \mathcal{L}(z) \rightarrow \mathcal{L}(z) \cup \mathcal{L}(y) \text { and } \\
\text { 2. if } z \text { is an ancestor of } x \\
\text { then } \mathcal{L}(\langle z, x\rangle) \longrightarrow \mathcal{L}(\langle z, x\rangle) \cup \operatorname{lnv}(\mathcal{L}(\langle x, y\rangle)) \\
\text { else } \mathcal{L}(\langle x, z\rangle) \longrightarrow \mathcal{L}(\langle x, z\rangle) \cup \mathcal{L}(\langle x, y\rangle) \\
\text { 3. } \mathcal{L}(\langle x, y\rangle) \longrightarrow \emptyset \text { and set } u \neq z \text { for all } u \text { with } u \neq y\end{array}$ \\
\hline$\left(\leq_{r_{\triangleright}}\right)$ & if 1. & $\begin{array}{l}\langle\leq p R . C, \triangleright, n\rangle \in \mathcal{L}(x), \\
\sharp R_{c}^{\mathcal{F}}(x, \triangleright, n, C)>p \text {, there are two of them } y, z \text {, both root nodes, with no } y \neq z \text { and } \\
\text { 1. } \mathcal{L}(z) \rightarrow \mathcal{L}(z) \cup \mathcal{L}(y) \text { and } \\
\text { 2. For all edges }\langle y, w\rangle \text { : } \\
\text { i. if the edge }\langle z, w\rangle \text { does not exist, create it with } \mathcal{L}(\langle z, w\rangle)=\emptyset \\
\text { ii. } \mathcal{L}(\langle z, w\rangle) \longrightarrow \mathcal{L}(\langle z, w\rangle) \cup \mathcal{L}(\langle y, w\rangle) \\
\text { 3. For all edges }\langle w, y\rangle \text { : } \\
\text { i. if the edge }\langle w, z\rangle \text { does not exist, create it with } \mathcal{L}(\langle w, z\rangle)=\emptyset \\
\text { ii. } \mathcal{L}(\langle w, z\rangle) \longrightarrow \mathcal{L}(\langle w, z\rangle) \cup \mathcal{L}(\langle w, y\rangle) \\
\text { 4. Set } \mathcal{L}(y)=\emptyset \text { and remove all edges to/from } y \text {, set } u \neq z \text { for all } u \text { with } u \neq y \text { and set } y \doteq z\end{array}$ \\
\hline
\end{tabular}

completion rules is applicable. The algorithm stops when a clash occurs; it answers ' $\mathcal{A}$ is consistent' iff the completion rules can be applied in such a way that they yield a complete and clash-free completion forest, and ' $\mathcal{A}$ is inconsistent' otherwise.

Lemma 4.6: Let $\mathcal{A}$ be an $\mathrm{f}_{K D^{-}} \mathcal{A} \mathcal{L C} \mathcal{C} \mathcal{Q}$ ABox. Then,

1) when started for $\mathcal{A}$ the tableaux algorithm terminates.

2) $\mathcal{A}$ has a fuzzy tableau if and only if the expansion rules can be applied to $\mathcal{A}$ such that they yield a complete and clash-free completion forest.

Proof: [sketch] The proof of this lemma follows the same principles as those shown for other more expressive fuzzy DLs like $\mathrm{f}_{K D}-\mathcal{S H \mathcal { H } N}[12]$.

Termination (first claim in Lemma 4.6) is a result of the properties of the expansion rules as in the classical case [24] and the case for fuzzy DLs shown in [12].

For the "if" case of the second claim, we can obtain a fuzzy tableau $T=(\mathbf{S}, \mathcal{L}, \mathcal{E}, \mathcal{V})$ from a complete and clash free completion-forest $\mathcal{F}$ quite trivially since in the absence of GCIs the algorithm terminates and thus no blocking is required [24], [12]. In brief let $\mathcal{F}$ be a complete and clashfree completion-forest constructed by the tableaux algorithm for $\mathcal{A}$. A fuzzy tableau $T=(\mathbf{S}, \mathcal{L}, \mathcal{E}, \mathcal{V})$ can be defined as follows:

$$
\begin{aligned}
& \mathbf{S}=\{x \mid x \text { is a node in } \mathcal{F}, \text { and } x\}, \\
& \mathcal{L}(x, \perp)=0, \text { for all } x \in \mathbf{S}, \\
& \mathcal{L}(x, \top)=1, \text { for all } x \in \mathbf{S}, \\
& \mathcal{L}(x, C)=g l b\left\{\left[\triangleright, n_{i}\right]\right\}, \text { for all } x \text { in } \mathcal{F}, \\
& \mathcal{L}(x, \neg A)= 1-\mathcal{L}(x, A), \text { for all } x \text { in } \mathcal{F}, \\
& \quad \text { with }\langle\neg A, \geq, n\rangle \in \mathcal{L}(x), \\
& \mathcal{E}(R,\langle x, y\rangle)=\left\{g l b\left\{\left[\geq, n_{i}\right]\right\} \mid y \text { an } R_{\geq n_{i}} \text {-successor of } x\right\} \\
& \mathcal{V}\left(a_{i}\right)=x_{0}^{i}, \text { where } x_{0}^{i} \text { is a root node }
\end{aligned}
$$

where $g l b$ is defined in [9] and returns the maximum degree $n$ out of the set of membership triples of the form $\left\langle A, \geq, n_{i}\right\rangle$, or $n+\epsilon$, where $\epsilon$ is a small real number converging to 0 , if the maximum degree appears in a membership triple of the form $\left.\langle A\rangle,, n_{i}\right\rangle$, or finally 0 if no such triple exists. It can be shown that $T$ is a fuzzy tableau for $\Sigma$.

For the "only-if" direction, following [12], if we have a 
fuzzy tableau $T$ for $\mathcal{A}$ we can use it to trigger the application of the expansion rules such that they yield a completionforest $\mathcal{F}$ that is both complete and clash-free.

Example 4.7: Lets see how our procedure can be used to identify the inconsistency of the ABox $\mathcal{A}_{c}$ we introduced in section III. Firstly, the algorithm initializes one root node $x_{a}$, labelled with $\mathcal{L}\left(x_{a}\right)=\{\langle\geq 3 R . \top, \geq, 0.7\rangle,\langle\leq 1 R . B, \geq$ $, 0.7\rangle,\langle\leq 1 R . \neg B, \geq, 0.7\rangle\}$. Then, we repeatedly apply the rules of Table III. First, the $\geq \geq$-rule creates three $b_{i}$, such that $\mathcal{L}\left(\left\langle x_{a}, b_{i}\right\rangle\right)=\{\langle R, \geq, 0.7\rangle\}, \mathcal{L}\left(b_{i}\right)=\{\langle\top, \geq, 0.7\rangle\}$ and $b_{1} \neq b_{2} \neq b_{3}$. Then, for the triple $\langle\leq 1 R . B, \geq, 0.7\rangle$ the choose-rule has to non-deterministically add either $\langle\neg B, \geq$ $, 0.7\rangle$, or $\langle B,>, 0.3\rangle$ in the labels $\mathcal{L}\left(b_{i}\right)$. For either of the choices we will get a clash:

1) If we choose the triple $\langle B,>, 0.3\rangle$ for two of them then: $\langle\leq 1 R . B, \geq, 0.7\rangle \in \mathcal{L}\left(x_{a}\right)$, and for two $b_{i}$, $\left\langle x_{a}, b_{i}\right\rangle$ is conjugated with $\langle\neg R, \geq, 0.7\rangle, \mathcal{L}\left(b_{i}\right) \cup$ $\{\langle\neg B, \geq, 0.7\rangle\}$ contains a clash and all $b_{i}$ are different with each other.

2) If we choose $\langle\neg B, \geq, 0.7\rangle$ for two of them then: $\langle\leq$ $1 R . B, \geq, 0.7\rangle \in \mathcal{L}\left(x_{a}\right)$, and for two $b_{i},\left\langle x_{a}, b_{i}\right\rangle$ is conjugated with $\langle\neg R, \geq, 0.7\rangle, \mathcal{L}\left(b_{i}\right) \cup\{\langle\neg \neg B, \geq, 0.7\rangle\}$ contains a clash (the added triple $\langle\neg B, \geq, 0.7\rangle$ clashes with $\langle B, \geq, 0.7\rangle \equiv\langle\neg \neg B, \geq, 0.7\rangle)$ and all $b_{i}$ are different with each other.

Thus, all possible expansions result to a clash and $\mathcal{A}_{c}$ is inconsistent.

Theorem 4.8: The tableaux algorithm is a decision procedure for the consistency of $\mathrm{f}_{K D}-\mathcal{A L C \mathcal { Q }}$ ABoxes and the satisfiability and subsumption of $\mathrm{f}_{K D}-\mathcal{A L C} \mathcal{L} \mathcal{Q}$-concepts with respect to simple and acyclic terminologies.

Theorem 4.8 is an immediate consequence of lemmas 4.3 and 4.6. Moreover, as we discussed in section II, subsumption can be reduced to consistency checking for ABoxes.

\section{COnClusions}

Today more and more applications are using Semantic Web technologies, in order to be able to publish their content on the Web. Unfortunately, many of these application have special knowledge representational needs, hence the adoption of Semantic Web languages, like OWL and DLs, in such domains and applications is difficult. More precisely, many applications need the ability to represent and reason about uncertain and fuzzy knowledge. To this extent we have presented an extension of the description logic $\mathcal{A L C I} \mathcal{Q}$ with fuzzy set theory. The fuzzy $\mathrm{DL} \mathrm{f}_{K D}-\mathcal{A L C \mathcal { Q }}$ extends the fuzzy DL $\mathrm{f}_{K D}-\mathcal{A L C}$ [9] with inverse roles and QCRs. Although QCRs are not part of OWL they will be added in the future since they are very important for practical applications [4]. Additionally, we have presented a detailed reasoning algorithm for deciding $\mathrm{f}_{K D}-\mathcal{A L C \mathcal { Q }}$ ABox consistency. In order to achieve this goal we have provided an investigation of the properties of fuzzy QCRs, in order to provide sound rules for such types of concept constructors.

As far as future directions are concerned, these will include the extension of the algorithm of $\mathrm{f}_{K D}-\mathcal{S H \mathcal { H } N}$ [12] with the algorithm for QCRs presented here and moreover the addition of nominals $(\mathcal{O})$. Thus, we plan to provide full reasoning for the DL language $\mathrm{f}_{K D^{-}} \mathcal{S H O} \mathcal{H} \mathcal{Q}$. Additionally, we also plan to provide a tableaux reasoning algorithm for fuzzy nominals $\left(\mathcal{O}_{f}\right)$ which have been proposed in [26] and for which no tableaux algorithm exists, thus providing reasoning for $\mathrm{f}_{K D}-\mathcal{S H O}{ }_{f} \mathcal{I} \mathcal{Q}$.

\section{ACKNOWLEDGEMENTS.}

This paper was partially supported by the European Commission under project X-Media (FP6-26978). The work of Giorgos Stoilos was partially supported by project PENED 03ED475 2003, which is cofinanced $75 \%$ of public expenditure through EC - European Social Fund, 25\% of public expenditure through Ministry of Development - General Secretariat of Research and Technology and through private sector, under measure 8.3 of OPERATIONAL PROGRAMME "COMPETITIVENESS" in the 3rd Community Support Programme.

\section{REFERENCES}

[1] Tim Berners-Lee, James Hendler, and Ora Lassila. The semantic web. Scientific American, 2001.

[2] Sean Bechhofer, Frank van Harmelen, James Hendler, Ian Horrocks, Deborah L. McGuinness, Peter F. Patel-Schneider, and Lynn Andrea Stein eds. OWL web ontology language reference. Technical report, Feb 2004.

[3] F. Baader, D. McGuinness, D. Nardi, and P.F. Patel-Schneider. The Description Logic Handbook: Theory, implementation and applications. Cambridge University Press, 2002.

[4] C. Golbreich, O. Dameron, B. Gibaud, and A. Burgun. Web ontology language requirements w.r.t. expressiveness of taxonomy and axioms in medicine. In 2nd International Semantics Web Conference (ISWC 03), pages 180-194, 2003.

[5] B. Neumann and R. Möller. On scene interpretation with description logics. In H.I. Christensen and H.-H. Nagel, editors, Cognitive Vision Systems: Samping the Spectrum of Approaches, number 3948 in LNCS, pages 247-278. Springer, 2006.

[6] R. Moeller, B. Neumann, and M. Wessel. Towards computer vision with description logics-some recent progress. In Workshop on Integration of Speech and Image Understanding, pages 101-115, 1999.

[7] Diego Calvanese, Giuseppe De Giacomo, Maurizio Lenzerini, Daniele Nardi, and Riccardo Rosati. Description logic framework for information integration. In Proc. of the 6th Int. Conf. on the Principles of Knowledge Representation and Reasoning (KR'98), pages 2-13, 1998.

[8] Stephan Tobies. Complexity Results and Practical Algorithms for Logics in Knowledge Representation. $\mathrm{PhD}$ thesis, Rheinisch-Westfälischen Technischen Hochschule Aachen, 2001. URL http://lat.inf.tudresden.de/research/phd/Tobies-PhD-2001.pdf .

[9] U. Straccia. Reasoning within fuzzy description logics. Journal of Artificial Intelligence Research, 14:137-166, 2001.

[10] S. Hölldobler, T. D. Khang, and H.-P. Störr. A fuzzy description logic with hedges as concept modifiers. In Proceedings InTech/VJFuzzy'2002, pages 25-34, 2002.

[11] Daniel Sanchez and Andrea G.B. Tettamanzi. Fuzzy quantification in fuzzy description logics. In Elie Sanchez, editor, Capturing Intelligence: Fuzzy Logic and the Semantic Web. Elsevier, 2006.

[12] Giorgos Stoilos, Giorgos Stamou, Vassilis Tzouvaras, Jeff Z. Pan, and Ian Horrocks. Reasoning with very expressive fuzzy description logics. Journal of Artificial Intelligence Research, 30(5):273-320, 2007.

[13] Giorgos Stoilos, Nikos Simou, Giorgos Stamou, and Stefanos Kollias. Uncertainty and the semantic web. IEEE Intelligent Systems, 21(5):8487, 2006.

[14] U. Straccia. Description logics with fuzzy concrete domains. In 21st Conf. on Uncertainty in Artificial Intelligence (UAI-05), Edinburgh, 2005.

[15] Fernando Bobillo and Umberto Straccia. A fuzzy description logic with product t-norm. In Proceedings of the IEEE International Conference on Fuzzy Systems (Fuzz-IEEE-07), London, 2007. 
[16] G. Stoilos, G. Stamou, V. Tzouvaras, J.Z. Pan, and I. Horrocks. The fuzzy description logic f-SHIN $\mathcal{N}$. In Proc. of the International Workshop on Uncertainty Reasoning for the Semantic Web, pages 6776, 2005.

[17] Yanhui Li, Baowen Xu, Jianjiang Lu, and Dazhou Kang. Reasoning technique for extended fuzzy $\mathcal{A L C} \mathcal{Q}$. In ICCSA (2), pages 1179-1188, 2006.

[18] G. Stoilos, U. Straccia, G. Stamou, and J.Z. Pan. General concept inclusions in fuzzy description logics. In Proceedings of the 17th European Conference on Artificial Intelligence (ECAI 06), pages 457461. IOS Press, 2006.

[19] U. Straccia. Towards a fuzzy description logic for the semantic web. In Proceedings of the 2nd European Semantic Web Conference, 2005.

[20] G. Stoilos, G. Stamou, and J.Z. Pan. Handling imprecise knowledge with fuzzy description logics. In Proceedings of the International Workshop on Description Logics (DL 2006), Lake District, UK, 2006.

[21] P. Hajek. Making fuzzy description logic more general. Fuzzy Sets and Systems, 154(1):1-15, 2005

[22] B. Hollunder and F. Baader. Qualified number restrictions in concept languages. In Proceedings of the 2nd International Conference on Principles of Knowledge Representation and Reasoning (KR'91), pages 335-346. Morgan Kaufman, 1991.

[23] Bernhard Hollunder, Werner Nutt, and Manfred Schmidt-Schaus. Subsumption algorithms for concept description languages. In European Conference on Artificial Intelligence, pages 348-353, 1990.

[24] I. Horrocks, U. Sattler, and S. Tobies. Reasoning with Individuals for the Description Logic $\mathcal{S H I} \mathcal{I}$. In David MacAllester, editor, $C A D E$ 2000, number 1831 in LNAI, pages 482-496. Springer-Verlag, 2000.

[25] Yanhui Li, Baowen Xu, Jianjiang Lu, and Dazhou Kang. Discrete tableau algorithms for fshi. In Proceedings of the International Workshop on Description Logics (DL 2006), Lake District, UK, 2006.

[26] Fernando Bobillo, Miguel Delgado, and Juan Gómez-Romero. A crisp representation for fuzzy SHOIN with fuzzy nominals and general concept inclusions. In Proc. of the 2nd International Workshop on Uncertainty Reasoning for the Semantic Web (URSW 06), Athens, Georgia, 2006. 